\title{
Seminal fluid gene expression and reproductive fitness in Drosophila melanogaster
}

\author{
Bahar Patlar and Alberto Civetta
}

\begin{abstract}
Background: The rapid evolution of seminal fluid proteins (SFPs) has been suggested to be driven by adaptations to postcopulatory sexual selection (e.g. sperm competition). However, we have recently shown that most SFPs evolve rapidly under relaxed selective pressures. Given the role of SFPs in competition for fertilization phenotypes, like the ability to transfer and store sperm and the modulation of female receptivity and ovulation, the prevalence of selectively relaxed SFPs appears as a conundrum. One possible explanation is that selection on SFPs might be relaxed in terms of protein amino acid content, but adjustments of expression are essential for post-mating function. Interestingly, there is a general lack of systematic implementation of gene expression perturbation assays to monitor their effect on phenotypes related to sperm competition.

Results: We successfully manipulated the expression of 16 SFP encoding genes using tissue-specific knockdowns (KDs) and determined the effect of these genes' perturbation on three important post-mating phenotypes: female refractoriness to remating, defensive (P1), and offensive (P2) sperm competitive abilities in Drosophila melanogaster. Our analyses show that KDs of tested SFP genes do not affect female refractoriness to remating and P2, however, most gene KDs significantly decreased P1. Moreover, KDs of SFP genes that are selectively constrained in terms of protein-coding sequence evolution have lower P1 than KDs of genes evolving under relaxed selection.
\end{abstract}

Conclusions: Our results suggest a more predominant role, than previously acknowledged, of variation in gene expression than coding sequence changes on sperm competitive ability in D. melanogaster.

Keywords: Seminal fluid proteins, Post-mating phenotypes, Sperm competition, Gene expression, RNAi knockdowns

\section{Background}

Male biased genes are known to be rapidly evolving, and their rapid evolution has been suggested to be driven by adaptations to reproductive functions [1-3]. Among them, seminal fluid proteins (SFPs) are recognized to be among those evolving the fastest $[1,4-8]$. However, both population genetics and molecular evolution studies have found a limited number of SFPs under positive directional selection $[4,7,9,10]$. A recent population

\footnotetext{
*Correspondence: a.civetta@uwinnipeg.ca

Department of Biology, University of Winnipeg, Winnipeg, MB R3B 2E9 Canada
}

genetics analysis of approximately 300 SFPs using data from a North American and an African population of Drosophila melanogaster found, depending on the population assayed, only $7-12 \%$ of all known SFPs evolving rapidly under positive selection. Among the remaining SFPs, $35-37 \%$ were selectively constrained and $50-57 \%$ were selectively relaxed [11].

Functional characterization of SFPs have shown them to be capable to affect reproduction in several ways such as sperm capacity, storage, and release [12, 13], stimulation of female egg production [14, 15], female postmating physiology and behaviour [16, 17], paternity success and sperm competition [18]. Given the functions 
of known SFPs in the reproductive success of males by assisting sperm in fertilization, as well as providing defensive and offensive advantages against rivals in sperm competition, the prevalence of relaxed selective pressures shaping the evolution of SFPs is somewhat surprising.

The apparent discrepancy between a large proportion of selectively relaxed SFPs and the known importance of SFPs in reproductive success highlights a long-standing problem of connecting the genotype to the phenotype in an attempt to understand their evolution [19]. This apparent phenotype-genotype disconnection could be attributed to different reasons. One possibility is that a limited number of SFPs under positive or purifying selection are sufficient to drive adaptations at the phenotypic level. This is likely to take place under a genetic architecture in which a handful of major genes exert main effects, with modulation by minor modifiers [20]. For example, in Drosophila melanogaster, the nuclear receptor HR39 has been shown as capable of regulating the expression of more than half of putative SFP encoding genes [21]. This genetic architecture predicts that mutations of minor SFP modifiers should have limited effects on fitness. A second possibility is that several SFPs are selectively relaxed because amino acid changes do not cause any modification of protein function (neutral), but changes in expression and the amount of product are essential for gene function and subjected to selective pressures. Indeed, SFPs are highly plastic in gene expression as a response to post-mating selective pressures such as sperm competition [22]. If changes in expression of SFP genes affect male fitness, understanding the effect of gene expression perturbation on fitness shall offer a better opportunity to establish links between the evolution of genotypes and phenotypes.

Drosophila females are known to mate multiply with a varying number of males $[23,24]$. Thus, while the effect of SFP gene deletions, nulls, and knockdowns in non-competitive mating experiments provide valuable information about gene function, testing the effect of perturbation of gene expression in a design that incorporates competitive mating is critical for an understanding of the role of differential gene expression in sperm competition. Support for a handful of SFPs affecting competitive fitness comes from independent studies that find associations between gene polymorphisms and phenotypes in competitive settings and those that test the effect of the same gene disruption on a phenotype of likely importance in competitiveness (e.g. sperm storage) [18]. There is a lack of systematic implementation of experimental competitive settings and the use of gene perturbation assays to monitor the effect of changes in gene expression on sperm competition phenotypes. A few exceptions are knockdowns of Acp36DE, CG17575,
CG9997, and SP affecting defensive (P1) and/or offensive (P2) sperm competitive ability $[25,26]$. Among these, SP, which is a functionally well-characterized SFP affecting female remating, has been shown as exhibiting high variation in gene expression level that is non-linearly related to female delay to remating [27].

Here we manipulated the expression of 20 SFP encoding genes using tissue-specific knockdowns and determined the effect of successful gene perturbations on important post-mating responses. Specifically, we assayed male fertility in single matings, male paternity success in competition (i.e. defensive and offensive sperm competitiveness; P1 and P2 respectively) [28, 29] and female refractoriness to remate. We selected SFP genes as candidates to test based on (i) evidence from the literature showing associations between SFPs polymorphisms and sperm competitiveness, (ii) SFPs having defined functions in non-competitive sperm physiology or fecundity based on studies that used gene nulls/knockdowns, and (iii) the availability of transgenic D. melanogaster strains for gene expression disruption. We also included the SFP gene Acp29AB to the list as an internal control, given that it is known to have a significant effect on P1 but not on female refractoriness and P2 [30]. In addition, amongst the genes successfully downregulated, seven genes showed rapid protein coding sequence evolution driven by relaxed selection and five conserved protein coding content due to selective constraints [11]. These genes allowed us to evaluate the effect of gene perturbation of SFPs expression in the context of different evolutionary histories at the protein sequence level.

\section{Results}

\section{RNAi knockdowns}

We tested the effectiveness of knockdowns by using qPCR to measure the expression of target genes (Table 1) in knockdown flies relative to wild-type controls. Out of the 20 SFP genes tested, $13 \mathrm{KDs}$ had significantly lower expression than control males $(P \leq 0.1$; Additional file 1 : Fig. S1). For another 3 genes (Semp1, msopa, Qsox4), expression could not be detected in the KDs (Additional file 1: Fig. S1), but the expression in the control was normal thus indicating nearly complete down-regulation. KD of another four genes (antr, intr, CG6168, Spn38F) did not result in a significant down-regulation of gene expression (Additional file 1: Fig. S1).

\section{Male fertility in single mating does not decrease in knockdowns}

Male fertility in single mating assays was scored to test for any significant impairment of the KDs that may affect their competitive paternity in double-mating experiments. Offspring numbers were counted at $1,2,3$, and 
Table 1 SFP genes selected to test their knockdown effect on male fertility, female refractoriness and sperm competitiveness

\begin{tabular}{|c|c|c|c|c|}
\hline Gene name & $\begin{array}{l}\text { Phenotypes from gene } \\
\text { perturbation assays* }\end{array}$ & $\begin{array}{l}\text { Genotype-phenotype } \\
\text { associations }\end{array}$ & Selection regime & References \\
\hline $\begin{array}{l}\text { Acp29AB } \\
(\text { CG17797) }\end{array}$ & P1, sperm storage & P1 & Relaxed/Positive & Clark et al. [61], Wong et al. [72] \\
\hline $\begin{array}{l}\text { Acp33A } \\
\text { (CG6555) }\end{array}$ & & P1, P2 & Relaxed & Fiumera et al. [63], Reinhart et al. [78] \\
\hline $\begin{array}{l}\text { Acp53Ea } \\
\text { (CG8622) }\end{array}$ & & P1 & Relaxed & Clark et al. [61] \\
\hline $\begin{array}{l}\text { Acp76A } \\
(\text { CG3801) }\end{array}$ & & P1, P2 & Constrained & Clark et al. [61], Fiumera et al. [60] \\
\hline $\begin{array}{l}\text { antr } \\
\text { (CG30488) }\end{array}$ & $\begin{array}{l}\text { SP activity, sperm storage, remating, } \\
\text { fecundity }\end{array}$ & & Positive & Findlay et al. [64], Singh et al. [65] \\
\hline $\begin{array}{l}\text { agrs } \\
\text { (CG14061) }\end{array}$ & $\begin{array}{l}\text { SP activity, sperm storage, remating, } \\
\text { fecundity }\end{array}$ & & Constrained & Findlay et al. [64], Singh et al. [65] \\
\hline CG11598 & $\begin{array}{l}\text { Mediating the activity of AG main } \\
\text { cells }\end{array}$ & & Constrained & Hopkins et al. [69] \\
\hline CG17242 & & $\begin{array}{l}\text { Correlated expression with } \\
\text { CG9997+ }\end{array}$ & Relaxed/Positive & Ayroles et al. [70] \\
\hline CG34002 & & Correlated expression with CG9997+ & Relaxed & Ayroles et al. [70] \\
\hline CG9168 & & DA in response to density & Constrained & Hopkins et al. [71] \\
\hline $\begin{array}{l}\text { msopa } \\
\text { (CG14560) }\end{array}$ & & Remating, P1, fecundity & - & $\begin{array}{l}\text { Fiumera et al. [60], Zhang et al. [62], } \\
\text { Reinhart et al. [78] }\end{array}$ \\
\hline $\begin{array}{l}\text { QSox4 } \\
\text { (CG31413) }\end{array}$ & & Correlated expression with CG9997 ${ }^{+}$ & Constrained & Ayroles et al. [70] \\
\hline $\begin{array}{l}\text { Semp1 } \\
\text { (CG11864) }\end{array}$ & $\begin{array}{l}\text { Normal processing of genes } \\
\text { Acp26A } 2 A^{+} \text {and } A c p 36 D E^{+}\end{array}$ & & Relaxed & $\begin{array}{l}\text { Ravi Ram et al. [79], LaFlamme et al. } \\
\text { [66] }\end{array}$ \\
\hline $\begin{array}{l}\text { Sems } \\
\text { (CG10586) }\end{array}$ & $\begin{array}{l}\text { Sperm storage and release, fecun- } \\
\text { dity }\end{array}$ & & Relaxed & LaFlamme et al. [66] \\
\hline $\begin{array}{l}\text { Sfp38D } \\
\text { (CG42606) }\end{array}$ & & DA in response to density & Relaxed & Hopkins et al. [71] \\
\hline $\begin{array}{l}\text { Spn28F } \\
\text { (CG8137) }\end{array}$ & & Remating, P1 & Positive & Fiumera et al. [63], Zhang et al. [62] \\
\hline $\begin{array}{l}\text { Spn77BC } \\
\text { (CG6289) }\end{array}$ & Spermatid individualization & & Relaxed & Kondo et al. [67] \\
\hline $\begin{array}{l}\text { Spn38F } \\
\text { (CG9334) }\end{array}$ & & Immune function in females & Relaxed & Mueller et al. [68] \\
\hline $\begin{array}{l}\text { intr } \\
\text { (CG12558) }\end{array}$ & $\begin{array}{l}\text { SP activity, sperm storage, remating, } \\
\text { fecundity }\end{array}$ & & Constrained & Findlay et al. [64], Singh et al. [65] \\
\hline CG6168 & & $\begin{array}{l}\text { Immune function in females, remat- } \\
\text { ing, P2 }\end{array}$ & Relaxed/Constrained & $\begin{array}{l}\text { Mueller et al. [68], Fiumera et al. [60], } \\
\text { Zhan g et al. [62] }\end{array}$ \\
\hline
\end{tabular}

AG accessory glands, $S P$ sex peptide, $D A$ differential abundance

+ Genes known to affect P1 and/or P2 (reviewed in Civetta and Ranz [18])

*All phenotypes, except for Acp29AB, tested in non-competitive assays

10 days after mating to resemble the intervals used in assays of competitive male paternity. We examined experimental block effects using a two-way ANOVA with days as covariates. There was a significant block effect on offspring counts for the control $\left(\mathrm{F}_{(1,171)}=30.95\right.$, $P<0.001)$, therefore we independently tested the fertility differences between KDs and the wild-type control within each experimental replica block. None of the tested males showed a significant decline in average cumulative offspring numbers compared to the control group (One-tailed Welch's $t$-tests; $P>0.05$ ) (Additional file 1: Table S2, Fig. 1).

\section{RNAi knockdown affects sperm competitive ability of first but not second males}

We evaluated defensive sperm competitive ability (P1) as short- and long-term relative paternity success (P1 in vial 2 and vial 3, respectively), and overall P1 (relative paternity success over vials 2 and 3 ). Our comparisons of KDs to the control group for overall P1 showed 


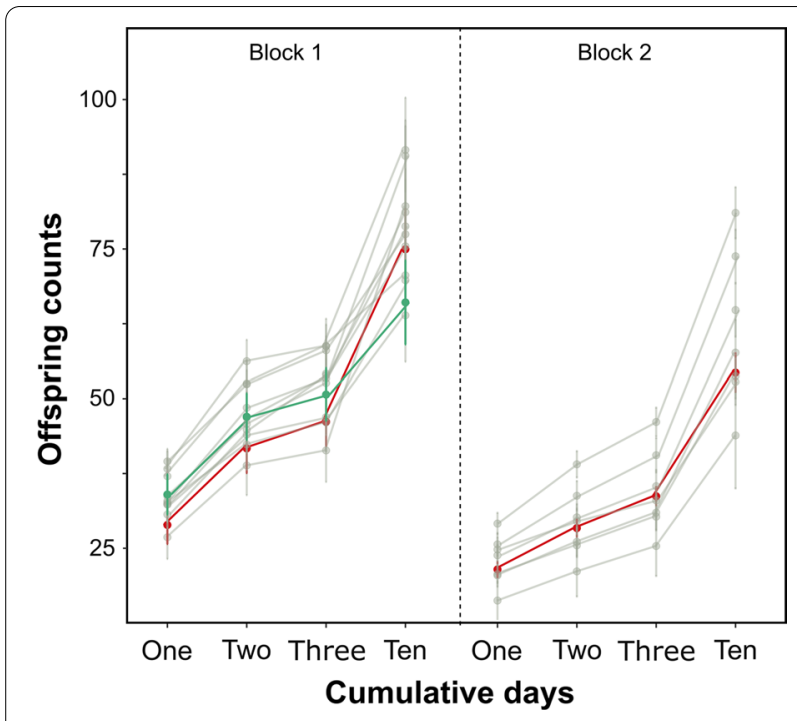

Fig. 1 Male fertility for experimental and control males. Mean cumulative number of offspring produced for the first 3 days and at 10 days after mating. Wild-type control (red), GFP reference (green) and SFP knockdowns (grey) are shown. Bars indicate standard errors of the means

significant declines in all KDs except for one gene, Sfp38D (Fig. 2A, Additional file 1: Table S3). The longterm success of all KDs in P1 (vial 3) was also significantly lower than the control group except for $S f p 38 D$ (Additional file 1: Table S3). Only eight KDs (Acp29AB, Acp53Ea, aqrs, CG11598, CG34002, CG9168, Qsox4, and Sems) had significant short-term (vial 2) decline in P1 (Additional file 1: Table S3). Temporal effects of KDs on sperm competitiveness (P1 and P2) have been previously reported [31, 32].

Differences in P1 between males can result from variation in the ability of first to mate males to interfere with the sperm from an incoming second male (e.g. male guardian or copulatory plugs, incapacitation of second male sperm). P1 can also be affected by differences in the properties and functioning of the first male sperm (e.g. differential sperm ejection by females, sperm entry and storage or sperm fertilization ability). Under the first scenario, high P1 values might not associate with the amount of progeny sired by the first male but rather with differences in progeny counts from the second male. We analysed the relationship between the total number of offspring sired by the first male and P1 to evaluate whether the differences in P1 reflect changes in the ability of KD males to assist first male sperm in competition. We found a highly significant regression between offspring number and P1 indicating that variation in P1 can be mostly explained by effects of the SFPs on the properties and functioning of the first male sperm (Adjusted $\mathrm{R}^{2}=0.6, P<0.001$; Additional file 1: Fig. S2).

Among the 16 genes that we effectively knockdown, the coding sequence evolution of 5 was previously identified as selectively constrained and 7 selectively relaxed [11]. We used these genes to evaluate whether there were differences in the effects of KDs on P1 (phenotype) based on the protein-coding sequences mode of evolution. A one-way ANOVA shows that selectively constrained genes as a group have substantially lower P1 values when their expression is knocked down than group of genes that evolved under relaxed selection (Fig. 2B; $\mathrm{F}_{(1,249)}=11$, $P=0.001$ ).

We found significant differences between the wild-type controls, run in the two separate blocks of P2 experiments, for all sperm competition intervals analysed $\left(\mathrm{F}_{(1,45) \text { Vial } 2}=16.7, \quad P<0.001 ; \mathrm{F}_{(1,45) \text { Vial } 3}=6.0, \quad P=0.02\right.$; $\left.\mathrm{F}_{(1,45) \text { Vial } 2+3}=12.8, P<0.001\right)$, therefore, results from the two blocks were analyzed separately. We found that all KDs, as well as the internal control gene Acp $29 A B$, were non-significantly lower in overall $\mathrm{P} 2$ when compared to control wild-type males (Fig. 2C, Additional file 1: Table S4). Short-term (vial 2) and long-term (vial 3) P2 estimates were also non-significant for all gene KDs tested (Additional file 1: Table S4).

\section{Mates of knockdown males showed no difference in remating compared to controls}

Some SFPs are known to work to make females refractory to remate. Therefore, we expected that at least some of our SFPs KDs would show an increase in remating rates relative to wild-type controls. We found no block effect within any of the days tested (Fisher's exact tests $P>0.05$ ), allowing us to pool the blocks for further comparisons. The proportion of females that remated with the reference GFP males after a single mating with a KD was not significantly different than the proportion that remated after mating with a wild-type control (Additional file 1: Table S5, Fig. 3). This result rules out possible effects on sperm competitiveness through manipulation of the females' mating behaviour.

\section{Discussion}

A handful of functions have been assigned to SFPs through studies that targeted them using gene perturbation and assayed the effect of such manipulation in non-competitive mating trials. The identified functions suggest a role of SFPs in increasing chances for paternity success in defensive situations, such as delay in female remating or enhancing sperm storage and retention [18]. In our study, we showed that down-regulation of 15 tested SFP genes dramatically decreased defensive first male paternity success (P1), while not affecting 

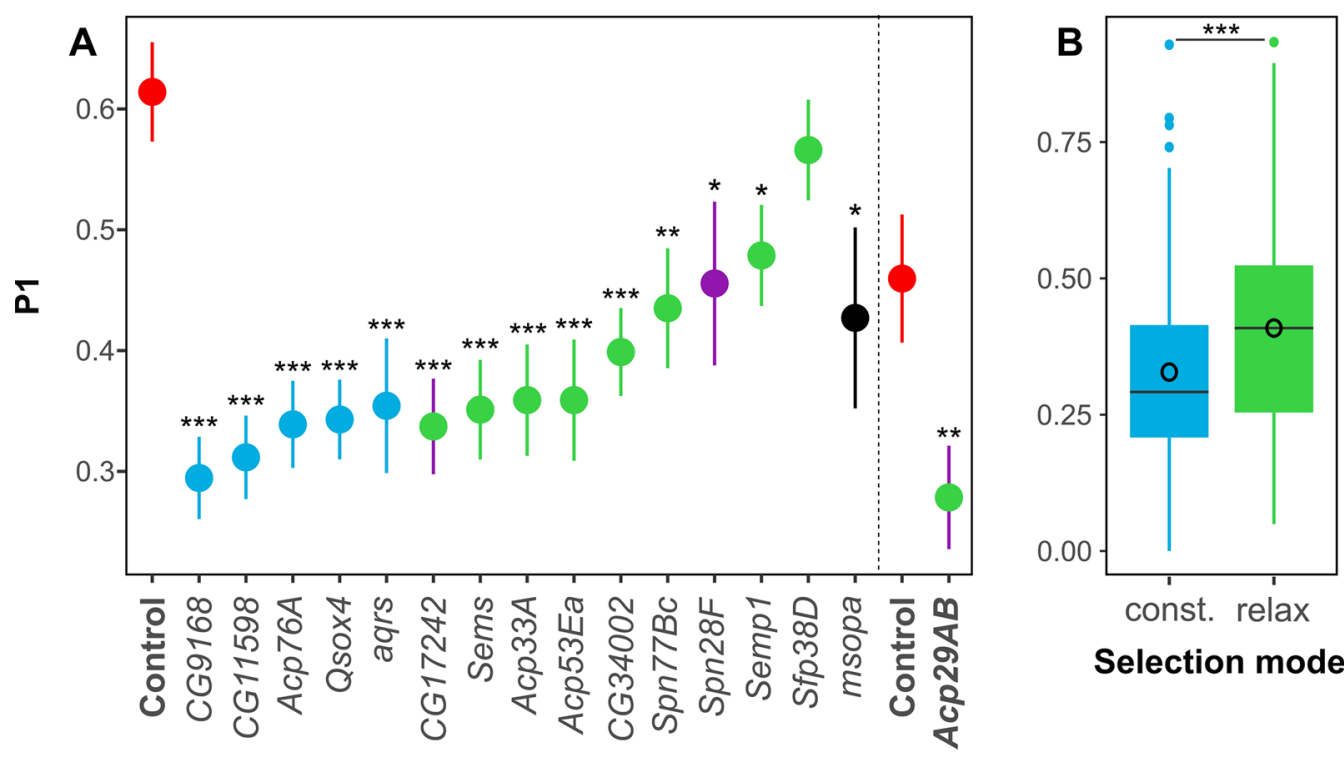

\section{Selection mode}

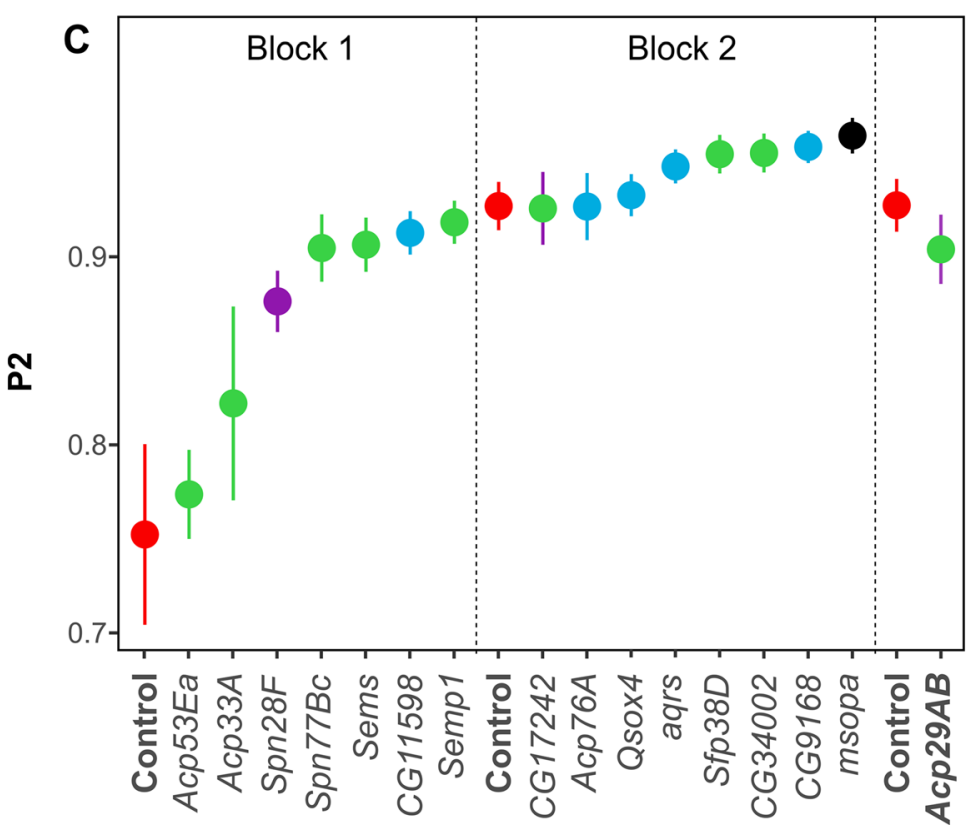

Fig. 2 Defensive (P1) and offensive (P2) sperm competitiveness of gene knockdowns and controls. Mean P1 (A) and P2 (C) for each KD (color coded) and wild-type control (red) are shown with error bars indicating the standard error of the means. Gene KDs are color coded based on their selection regime (Blue: selectively constrained, Green: Relaxed purifying selection, Purple: Positive selection, Black: Unknown). Two genes show both green and purple because they had population specific selection regimes. B Boxplot showing differences in overall effect of KDs on P1 for selectively constrained and relaxed genes. The mean and median P1 values are shown as circles and lines within the box, respectively. ${ }^{* * *} P<.001$, ${ }^{*} P<.01,{ }^{*} P<.05$

female refractoriness to remate and second male paternity success (P2). We interpret these results as evidence that SFPs can be more important than previously acknowledged in affecting the ejaculate ability to defend against rival sperm. Moreover, we have recently shown that the protein sequence of the majority of transferred SFPs has evolved under a pattern of relaxed purifying selection [11]. Therefore, our results suggest that gene regulation of SFPs could be an important factor affecting sperm competition phenotypes and that modifications in the amount of SFP produced, rather 


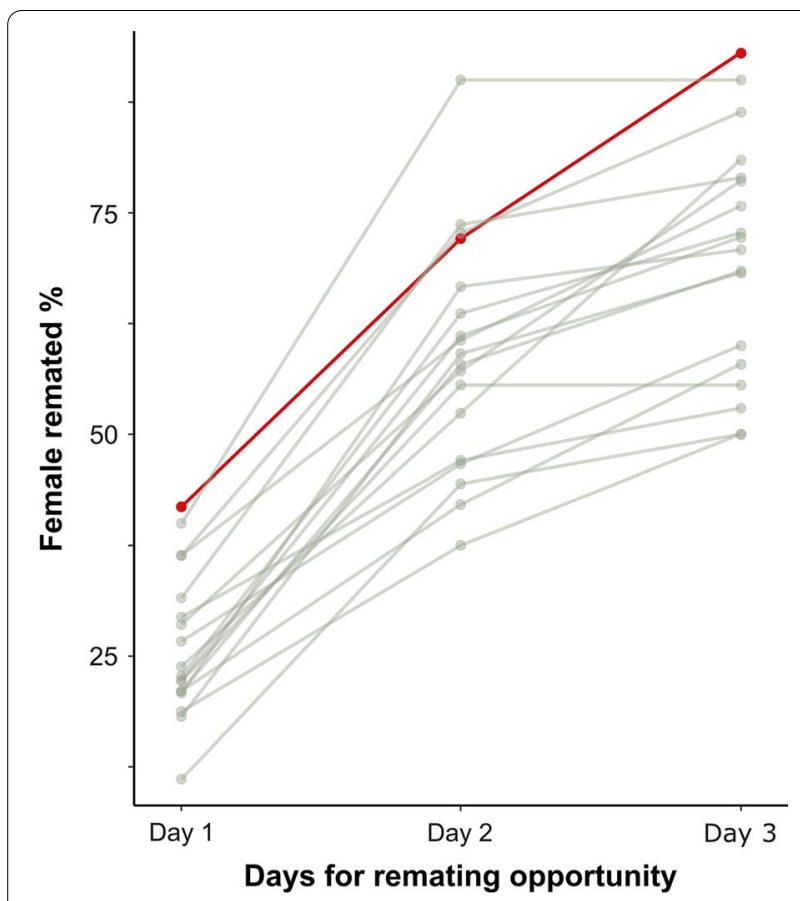

Fig. 3 Female refractoriness to remating. The percentage of females that remated with a GFP reference males within 3 days after their first mating with experimental KDs (grey lines) or wild-type control (red line) males

than changes in the protein itself, might contribute significantly to the evolution of SFP genes.

The last male advantage has been extensively documented in Drosophila melanogaster suggesting that defending sperm against upcoming rivals is a critical factor affecting male fitness [24, 33, 34]. We showed that defensive sperm competitive ability (P1) was dramatically decreased in SFPs KDs while male fertility and offensive sperm competitive ability (P2) were unaffected. The block effects we detected in male fertility and P2 tests are not surprising as such effects have been previously found in sperm competition trials and likely result from a myriad of environmental effects $[35,36]$. Importantly, the outcome of the gene perturbation effects relative to wildtype controls did not change among blocks.

A male's sperm defense could be affected by several factors, such as female remating time, sperm storage, ejection, retention, and release. For example, a previous study using null mutant flies characterized the function of the seminal fluid lectin Acp29AB. The absence of Acp29AB strongly decreased $\mathrm{P} 1$, but not $\mathrm{P} 2$, when females remated 2 days after their first mating. Moreover, females mated with Acp29AB null males were no different in remating rate or fertility. The study showed that Acp $29 A B$ is critical in sperm defense because it is needed for sperm retention in storage [30]. Acp36DE nulls affect sperm storage and decrease males' P1. Interestingly, the effect of Acp36DE nulls on P2 is a consequence of the inability of the sperm of Acp36DE nulls to be retained in storage, and not because Acp36DE is involved in the displacement of resident sperm $[25,37,38]$. There is a limited number of studies that have specifically assayed the effect of gene perturbation of SFPs in competitive settings, but the majority, even when non-competitive settings have been used, identify effects of SFPs on aspects of sperm function such as entry into storage, retention, and release and not in offensive displacement [18]. Our work shows, in a survey of multiple SFPs, that almost all SFPs tested are needed for first male paternity success while not affecting the ability of the males to father progeny when second to mate. We did not test what aspects of sperm competition is disrupted in these knockdowns, but the KDs did not show significant changes in female remating or fertility, suggesting that the decline in P1 is less likely related to changes in female mating behaviour or sperm fertilization capability (i.e. sperm numbers/quality). It could be argued that if roles related to sperm entry, storage and retention are in fact affected in these KDs, P2 should also have been lower for these males. However, the lack of effect in second mating might be explainable if the presence of first wild-type male SFPs in the female is able to help the second KD incoming sperm. A beneficial, rather than detrimental, effect of residual seminal fluids on the sperm of subsequent males has been previously shown in D. melanogaster $[39,40]$.

There are potential limitations to the conclusions we draw about the importance of changes in SFPs expression and P1 that are important to consider. One such limitation is that, for logistic reasons, we compared KDs to a single wild-type control and we used a single reference (GFP) competitor, therefore we lack information on polymorphism and effects on sperm competitiveness. Moreover, we cannot fully rule out the possibility that differences in genetic background of the KD strains might have differentially affected variables like time of maturity or viability that could influence P1 scores. However, we believe such effects are unlikely, as they would have also affected offensive sperm competitiveness (P2). Finally, the use of an AG-specific driver to KD the expression of our target genes might have resulted in an inability to detect effects on reproductive fitness mediated by the genes expression in other tissues. This is highly unlikely for the only gene that did not show any effect on reproductive fitness (Sfp38D) because this gene is AG enriched with almost no expression in other tissues (FlyAtlas2). However, some of the SFPs genes we targeted might exert additional effects on reproductive fitness through their lower but noticeable level of expression in other tissues. For example, some of the genes targeted in our study are 
reported as having low but detectable expression in the female brain (FlyAtlas2) and it is known that females can influence sperm utilization and both offense and defence sperm competition outcomes [31, 41-43].

The significant effect on phenotype (P1) we observed as driven by the modulation of expression of different SFPs is interesting in the context of the potential role of gene expression in fitness. Previous studies that have not directly manipulated gene expression have also suggested an important role of the amount of SFP gene products in fitness. For example, studies that have modified density to increase the risk and the intensity of sperm competition have found that competitive settings lead to significant adjustment of SFPs expression [44-47]. P1 has been shown to be more sensitive to environmental variation (i.e. normal atmosphere vs. high $\mathrm{CO}_{2}$ concentration) than P2 in Drosophila melanogaster [48]. Moreover, variation of SFP gene expression generated through genotype-by-environment interactions in competitive and non-competitive settings found a positive relationship between gene expression and P1 but not P2 in flatworms [49]. The use of experimental evolution to enforce monogamy in Drosophila has also shown that monogamous males become weaker sperm competitors and have lower expression of SFPs, but do not differ from polygamous males in their ability to diminish female remating [50]. Proteomics has also identified differences in protein expression and abundance of the seminal fluid of primates with different mating systems, with enrichment of proteins with putative roles in the formation of the copulatory plug [51]. Overall, the effect of differential SFPs gene expression in postcopulatory reproductive fitness might be more important than previously acknowledged.

How these changes in expression might shape the longterm evolution of post-mating phenotypes is yet unclear. It is interesting that SFPs we previously identified [11] as evolutionary constrained at the protein sequence level have a more drastic effect on P1, than evolutionary relaxed SFPs, when knockdown. This suggests a positive correlation between protein-coding evolution and gene expression divergence [52-55]. However, studies that have used divergent populations or subspecies have failed to find associations $[56,57]$. Moreover, while interesting, we only had a small number of SFPs to compare and to properly establish associations between protein coding mode of evolution and expression of SFPs.

The shifts in expression we induced through gene perturbation are likely detrimental for males because they dramatically lower their fitness (lower P1) relative to wild-type males. Our current result on the effect of changes in expression in fitness, together with our previous identification of a considerable proportion of SFPs with constrained protein sequence evolution [11], raises the question of whether shifts involving relaxation of purifying selection follow by resets of new species-specific patterns of purifying selection might be more relevant than previously considered during the evolution of SFPs. It also remains to be determined whether the genes we have perturbed and assayed in intraspecific settings are also important in the establishment of postcopulatory barriers between species. If so, the finding would add to previous evidence of sperm competition genes contributing to post-mating barriers between species [26, 32, $58]$ and support the possibility of speciation via sexual selection.

\section{Materials and methods Drosophila melanogaster stocks and maintenance}

An Ovulin-Gal4 driver stock was kindly provided by Dr. M. F. Wolfner (Cornel University, NY, US). Genespecific UAS-RNAi lines for 20 genes were purchased from the Vienna Drosophila Resource Center (VDRC) or the Bloomington Drosophila Stock Center (BDSC). These UAS-RNAi lines are from four different strains (Additional file 1: Table S1) and have different genotypic backgrounds. Wild-type flies were from an isofemale line derived from flies collected in Winnipeg, Manitoba (Canada). A stock that expresses the jellyfish green fluorescent protein (GFP) in the ocelli was purchased from the BDSC (BDSC32170; genotype $\left.\mathrm{w}^{[*}\right]$; $\mathrm{PBac}\{\mathrm{w}[+\mathrm{mW}$. hs $]=$ GreenEye.UASdsRed $\{$ Dmel1). Males from this stock were used in competitive paternity assays to identify offspring sired by the common reference GFP male from non-GFP experimental males. Throughout the experiments, flies were kept in either $8 \mathrm{oz}$. bottles containing $50 \mathrm{ml}$ of cornmeal-yeast-agar-molasses medium $($ CYAM) or in $27 \times 93 \mathrm{~mm}$ (diameter $\times$ height) vials containing $6-8 \mathrm{ml}$ of medium. Stocks were mantained in a 12:12-h light-dark cycle at $22 \pm 1{ }^{\circ} \mathrm{C}$. Flies were anesthetized using $\mathrm{CO}_{2}$ when necessary, but to avoid anaesthetic effects on fitness [59], $\mathrm{CO}_{2}$ was never used $24 \mathrm{~h}$ prior to experiments. We performed all experiments with three to 5 days old flies.

\section{Gene selection and RNAi knockdowns}

We surveyed the literature to identify candidate SFP genes (Table 1). We first included SFP genes having associations between SNPs and female remating, P1 and/or P2 (Acp53Ea, Acp33A, Acp76A, CG6168, msopa, Spn28F) [60-63]. Second, we included genes having functions in sperm physiology, fecundity or remating based on the effect of knockdowns/nulls or gene ectopic expression (antr, aqrs, CG11598, intr, Semp1, Sems, Spn77Bc, Spn38F) [64-69]. We also chose three genes (CG17242, CG34002 and QSox4) that are genetically correlated in their expression 
with CG9997, a gene known to affect sperm competitiveness [70]. CG9168 and Sfp38D have no known molecular function, but were included because their abundance is dependent on sperm competition intensity [71]. Finally, we also added a control, $A c p 29 A B$, known to affect P1 [72].

We used an accessory gland tissue specific driver (Ovulin-Gal4) that effectively drives accessory glandspecific expression of target genes in young adult males [73], and all the targeted genes are genes with highly enriched expression in the accessory glands or AG-specific genes (Flyatlas2). To knockdown (KD) expression of these candidate genes, virgin males from UAS-RNAi lines were introduced with virgin females from the Ovulin-Gal4 driver strain. Each candidate gene knockdown was replicated in 3-4 vials containing 5 females and 5 males each. To control for larval density, females and males were discarded 5 days after mating. $F_{1}$ virgin males were collected from these replicates, pooled and kept in controlled numbers of 10-15 flies per vial. For verification of the knockdowns, males were chosen randomly from replicates and the male reproductive tract dissected in $1 \times$ PBS. For each candidate gene, three RNA biological replicates were prepared from KDs and wild-type control males raised under the same conditions. RNA was isolated by using the Bio-Rad Aurum Total RNA Mini Kit and complementary DNA (cDNA) was synthesized from $1 \mathrm{ml}$ of RNA solution using iScript Select cDNA Synthesis Kit (Bio-Rad, CA, USA) following the manufacturer's instructions. Gene expression was quantified using a BioRad CFX96 Real-Time PCR Detection System. The reactions were performed using the iQ SYBR Green quantitative real-time PCR kit (Bio-Rad, CA, USA). Reaction volumes were set at $10 \mu \mathrm{l}$, containing $4 \mu \mathrm{l}$ iQ SYBR Green Supermix Kit, $150 \mathrm{nM}$ of each primer pair $(1.5 \mu \mathrm{l}$ per pair), $2 \mu \mathrm{l}$ nuclease-free water and $1 \mu \mathrm{l} \mathrm{cDNA}$. Initial thermal cycling conditions were 1 cycle at $95{ }^{\circ} \mathrm{C}$ for $5 \mathrm{~min}$, followed by 39 cycles of denaturation at $95{ }^{\circ} \mathrm{C}$ for $15 \mathrm{~s}$ and annealing at $59^{\circ} \mathrm{C}$ for $30 \mathrm{~s}$. Raw Ct values of replicates were obtained from the CFX Software (version 3.0). We designed primers (Additional file 1: Table S1) for each candidate gene using Primer3web version 4.1.0. We have previously tested different reference genes and found that the ribosomal protein $R p S 18$ had consistently the highest expression and least variability in expression between serial dilution replicas [74]. Thus, we used $R p S 18$ as a reference gene to normalize gene expression. The expression level of the target gene in each sample was determined by calculating $\Delta \mathrm{Cq}$ (Cycle quantification) as the $\mathrm{Cq}$ of the reference gene (RpS18) minus the Cq of the target gene [75].

\section{Male fertility}

Male fertility was measured as the number of offspring fathered by a focal male singly-mated to a virgin wildtype female. The male fertility assays examined whether GFP reference males and knockdowns of SFP genes produced less offspring than wild-type control males. Wildtype females were collected within 3-4 h after emergence and kept in groups of ten for 3-5 days. One day before mating, we housed females singly in freshly prepared vials containing CYAM fly medium and the next morning they were introduced to one same-aged KD, wild-type or GFP male. Pairs were watched until mating occurred for up to three hours. Immediately after mating ended, males were removed, and females were retained for $24 \mathrm{~h}$ in the vials to let them lay eggs. We transferred females daily to fresh vials every morning for 3 days (Days $1-3$ ) and then on Day 7, with all females being discarded on Day 10 as female fertility starts to diminish 10 days after mating [76]. We scored offspring and used data to estimate early (Days 1-3) and cumulative (Day 10) male fertility. Male fertility assays were performed in two subsequent blocks, under the exact same conditions, each consisting of the control group (wild-type males) and a subset of KDs.

\section{Defensive (P1) and offensive (P2) sperm competitive ability} We estimated sperm competitiveness as the proportion of progeny sired by the tested male. A "defense" (P1) and "offense" (P2) score was estimated for each knockdown and control group using GFP males as rivals [18]. In the P1 experiment, we housed virgin females with either KD or control males in bottles containing fresh food (ca. 25-30 individuals from each sex) for about $24 \mathrm{~h}$. After $24 \mathrm{~h}$, we discarded males and transferred females into single vials (Vial 1), where they remain for 2 days to recover mating receptivity. After 2 days, we transferred single females to fresh vials (Vial 2) and introduced one GFP male. GFP males were kept in the Vial 2 for $24 \mathrm{~h}$ before being discarded. Females laid eggs in Vial 2 for 2 more successive days and were then transferred to Vial 3 for an additional 4 days. We followed the exact same procedure to measure P2, with tested males (i.e. KDs and wild-type control) being second to mate with females already mated to GFP males. For logistic reasons, we performed P2 experiment in two blocks each containing the control group and a subset of KDs. In addition, both P1 and P2 experiments were conducted separately for the internal control gene, $A c p 29 A B$.

Females that produced no progeny in Vial 1 were deemed unsuccessful in their first mating and discarded from further analysis. We then counted number of offspring with GFP expression in their ocelli, and those with wild type eyes, using a fluorescent light source under a Nikon SMZ645 dissecting microscope. P1 and P2 were 
estimated as the proportion of tested males progeny over vials 2 and 3 (overall estimates) as well as in each vial separately (short and long term).

\section{Female refractoriness to remate}

Refractoriness to remate was estimated as the proportion of females that do not remate after a first mating, within a given period of time (24, 48 and $72 \mathrm{~h}$ ). To estimate refractoriness, virgin wild-type females were first collected and grown under the same conditions as those used in the fertility experiment. Females kept singly housed in vials for $24 \mathrm{~h}$, were presented in the morning with one experimental male (KDs or wild-type control). We observed the pairs until a mating took place or for a maximum of three hrs, at which point males were discarded and females kept in their vials. On the next morning (Day 1), we introduced singly mated females to GFP reference males, and any remating episode was monitored for 7-8 h. Females that did not mate, were given the opportunity to remate with a tester male on subsequent days (Days 2 and 3). The refractoriness experiment was performed in two blocks, under the exact same conditions, each consisting of the control group (wild-type males) and a subset of KDs. For each KD group and control, we counted the number of females remated and not remated to a second GFP male in each day for 3 days in total.

\section{Statistical analyses}

All data was analysed using R-software (v. 4.0.3; [77]). For fertility, P2 and refractoriness, we performed assays in two blocks, with each block consisting of a set of KDs and wild-type control. We first evaluated differences for the estimated phenotypes of controls among the blocks using one-way ANOVA. The data were pooled, if controls did not significantly differ from each other. The knockdown effect on gene expression, fertility and sperm competitiveness (P1 and P2) were assessed using a one-tailed Welch's $t$-test $\left(\mathrm{H}_{0}: \mu_{\text {knockdown }}<\mu_{\text {control }}\right)$. Refractoriness was tested using a $2 \times 2$ Fisher Exact tests with KD vs. control as rows and mated or non-mated as columns. $P$-values were adjusted to control for false positives, given multiple tests, using the Benjamini-Hochberg procedure (threshold $\alpha=0.05$ ).

\section{Supplementary Information}

The online version contains supplementary material available at https://doi. org/10.1186/s12862-022-01975-1.

Additional file 1: Figure S1. RNAi knockdown efficacy. Figure S2. The total number of offspring sired by the first male and its defensive sperm competitiveness, P1. Table S1. Gene-specific UAS-RNAi lines for 20 genes and primers used to test the effect of ovulin driven (ovU-GAL4) downregulation in the accessory glands. Table S2. Male fertility tests. Table S3. Sperm competitiveness as "defense" ability (P1) of knockdown versus wild-type control males. Table S4. Sperm competitiveness as "offense" ability (P2) of knockdown versus wild-type control males. Table S5.

Female refractoriness tests.

\section{Acknowledgements}

We would like to thank Prof. M. Wolfner from Cornell University, Ithaca, United States for providing Ovulin-driver Drosophila stock used in this study.

\section{Authors' contributions}

BP performed the experiments and analysed data with the contribution of AC. BP and AC contributed in writing the manuscript. Both authors read and approved the final manuscript.

\section{Funding}

This work was supported by an NSERC Discovery Grant (RGPIN-2017-04599) to $A C$.

\section{Availability of data and materials}

The datasets generated and/or analyzed during the current study are available in the University of Winnipeg Dataverse repository, at https://doi.org/10.5683/ SP3/TBECSY.

\section{Declarations}

Ethics approval and consent to participate

Not applicable.

\section{Consent for publication}

Not applicable.

\section{Competing interests}

The authors declare that they have no competing interests.

Received: 28 October 2021 Accepted: 15 February 2022

Published online: 23 February 2022

\section{References}

1. Swanson WJ, Vacquier VD. The rapid evolution of reproductive proteins. Genetics. 2002;3:137-44.

2. Clark NL, Aagaard JE, Swanson WJ. Evolution of reproductive proteins from animals and plants. Reproduction. 2006;131:11-22.

3. Turner LM, Hoekstra HE. Causes and consequences of the evolution of reproductive proteins. Int J Dev Biol. 2008;52:769-80.

4. Swanson WJ, Clark AG, Waldrip-Dail HM, Wolfner MF, Aquadro CF. Evolutionary EST analysis identifies rapidly evolving male reproductive proteins in Drosophila. Proc Natl Acad Sci USA. 2001;98:7375-9.

5. Haerty W, Jagadeeshan S, Kulathinal RJ, Wong A, Ram KR, Sirot LK, et al. Evolution in the fast lane: rapidly evolving sex-related genes in Drosophila. Genetics. 2007;177:1321-35.

6. Wilburn DB, Swanson WJ. From molecules to mating: rapid evolution and biochemical studies of reproductive proteins. J Proteomics. 2016;135:12-25.

7. Rowe M, Whittington E, Borziak K, Ravinet M, Eroukhmanoff F, Sætre GP, et al. Molecular diversification of the seminal fluid proteome in a recently diverged Passerine species pair. Mol Biol Evol. 2020;37:488-506.

8. Dean MD, Good JM, Nachman MW. Adaptive evolution of proteins secreted during sperm maturation: an analysis of the mouse epididymal transcriptome. Mol Biol Evol. 2008;25:383-92.

9. Begun D, Whitley P, Todd B, Waldrip-Dail H, Clark A. Molecular population genetics of male accessory gland proteins in Drosophila. Genetics. 2000;156:1879-88.

10. Clark NL, Swanson WJ. Pervasive adaptive evolution in primate seminal proteins. PLoS Genet. 2005;1:335-42.

11. Patlar B, Jayaswal V, Ranz JM, Civetta A. Nonadaptive molecular evolution of seminal fluid proteins in Drosophila. Evolution. 2021;75:2102-13. 
12. Poiani A. Complexity of seminal fluid: a review. Behav Ecol Sociobiol. 2006;60:289-310.

13. Avila FW, Sirot LK, LaFlamme B, Rubinstein CD, Wolfner MF. Insect seminal fluid proteins: identification and function. Annu Rev Entomol. 2011;56:21-40

14. Heifetz Y, Lung O, Frongillo EA, Wolfner MF. The Drosophila seminal fluid protein Acp26Aa stimulates release of oocytes by the ovary. Curr Biol. 2000;10:99-102

15. Heifetz $Y$, Tram $U$, Wolfner MF. Male contributions to egg production: the role of accessory gland products and sperm in Drosophila melanogaster. Proc R Soc Lond B. 2001;268:175-80.

16. Schjenken JE, Robertson SA. The female response to seminal fluid. Physiol Rev. 2020;100:1077-117.

17. Gillott C. Male accessory gland secretions: modulators of female reproductive physiology and behavior. Annu Rev Entomol. 2003;48:163-84.

18. Civetta A, Ranz JM. Genetic factors influencing sperm competition. Front Genet. 2019;10:820.

19. Lewontin RC. The genetic basis of evolutionary change. New York: Columbia University Press; 1974.

20. Carson HL, Templeton AR. Genetic revolutions in relation to speciation phenomena: the founding of new populations. Annu Rev Ecol Syst. 1984;15:97-132.

21. Praggastis SA, Nam HJ, Lam G, Child VIMB, Castillo DM, Thummel CS Regulation of male fertility and accessory gland gene expression by the Drosophila HR39 nuclear receptor. Dev Biol. 2021;479:51-60.

22. Ramm SA. Seminal fluid and accessory male investment in sperm competition. Philos Trans R Soc B. 2020;375:20200068.

23. Imhof M, Harr B, Brem G, Schlötterer C. Multiple mating in wild Drosophila melanogaster revisited by microsatellite analysis. Mol Ecol. 1998;7:915-7.

24. Harshman LG, Clark AG. Inference of sperm competition from broods of field-caught Drosophila. Evolution. 1998;52:1334-41.

25. Avila FW, Wolfner MF. Acp36DE is required for uterine conformational changes in mated Drosophila females. Proc Natl Acad Sci USA. 2009;106:15796-800.

26. Castillo DM, Moyle LC. Intraspecific sperm competition genes enforce post-mating species barriers in Drosophila. Proc R Soc B. 2014; 281:20142050.

27. Smith DT, Hosken DJ, Ffrench-Constant RH, Wedell N. Variation in sex peptide expression in D. melanogaster. Genet Res (Camb). 2009;91:237-42.

28. Parker GA. Sperm competition and its evolutionary consequences in the insects. Biol Rev. 1970;45:525-67.

29. Parker GA. Conceptual developments in sperm competition: a very brief synopsis. Philos Trans R Soc B. 2020;375:20200061.

30. Wong A, Albright SN, Giebel JD, Ravi Ram K, Ji S, Fiumera AC, et al. A role for Acp29AB, a predicted seminal fluid lectin, in female sperm storage in Drosophila melanogaster. Genetics. 2008;180:921-31.

31. Chen DS, Delbare SYN, White SL, Sitnik J, Chatterjee M, DoBell E, et al. Female genetic contributions to sperm competition in Drosophila melanogaster. Genetics. 2019;212:789-800.

32. Civetta A, Finn S. Do candidate genes mediating conspecific sperm precedence affect sperm competitive ability within species? A test case in Drosophila. G3 Genes Genomes Genet. 2014;4:1701-7.

33. Scott D, Williams E. Sperm displacement after remating in Drosophila melanogaster. J Insect Physiol. 1993;39:201-6.

34. Morrow EH, Stewart AD, Rice WR. Patterns of sperm precedence are not affected by female mating history in Drosophila Melanogaster. Evolution 2005;59:2608.

35. Fricke C, Bretman A, Chapman T. Adult male nutrition and reproductive success in Drosophila melanogaster. Evolution. 2008;62:3170-7.

36. Levesque L, Brouwers B, Sundararajan V, Civetta A. Third chromosome candidate genes for conspecific sperm precedence between $D$. simulans and D. mauritiana. BMC Genet. 2010:11:1-12.

37. Chapman T, Neubaum DM, Wolfner MF, Partridge L. The role of male accessory gland protein Acp36DE in sperm competition in Drosophila melanogaster. Proc R Soc B. 2000;267:1097-105.

38. Avila FW, Wolfner MF. Cleavage of the Drosophila seminal protein Acp36DE in mated females enhances its sperm storage activity. J Insect Physiol. 2017;101:66-72.

39. Holman L. Drosophila melanogaster seminal fluid can protect the sperm of other males. Funct Ecol. 2009;23:180-6.
40. Misra S, Wolfner MF. Drosophila seminal sex peptide associates with rival as well as own sperm, providing sp function in polyandrous females. eLife. 2020;9:1-21.

41. Clark AG, Begun DJ. Female genotypes affect sperm displacement in Drosophila. Genetics. 1998;149:1487-93.

42. Manier MK, Belote JM, Berben KS, Novikov D, Stuart WT, Pitnick S. Resolving mechanisms of competitive fertilization success in Drosophila melanogaster. Science. 2010;328:354-7.

43. Lüpold S, Pitnick S, Berben KS, Blengini CS, Belote JM, Manier MK. Female mediation of competitive fertilization success in Drosophila melanogaster. Proc Natl Acad Sci USA. 2013;110:10693-8.

44. Patlar B, Weber M, Ramm SA. Genetic and environmental variation in transcriptional expression of seminal fluid proteins. Heredity. 2019:122:595-611.

45. Sloan NS, Lovegrove M, Simmons LW. Social manipulation of sperm competition intensity reduces seminal fluid gene expression. Biol Lett. 2018;14:20170659.

46. Nakadera Y, Giannakara A, Ramm SA. Plastic expression of seminal fluid protein genes in a simultaneously hermaphroditic snail. Behav Ecol. 2019:30:904-13.

47. Fedorka KM, Winterhalter WE, Ware B. Perceived sperm competition intensity influences seminal fluid protein production prior to courtship and mating. Evolution. 2011;65:584-90.

48. Dobler R, Reinhardt K. Heritability, evolvability, phenotypic plasticity and temporal variation in sperm-competition success of Drosophila melanogaster. J Evol Biol. 2016;29:929-41.

49. Patlar B, Ramm SA. Genotype-by-environment interactions for seminal fluid expression and sperm competitive ability. J Evol Biol. 2020;33:225-36.

50. Hollis B, Koppik M, Wensing KU, Ruhmann H, Genzoni E, Erkosar B, et al. Sexual conflict drives male manipulation of female postmating responses in Drosophila melanogaster. Proc Natl Acad Sci USA. 2019;116:8437-44.

51. Claw KG, George RD, MacCoss MJ, Swanson WJ. Quantitative evolutionary proteomics of seminal fluid from primates with different mating systems. BMC Genomics. 2018;19:1-17.

52. Lemos B, Bettencourt BR, Meiklejohn CD, Hartl DL. Evolution of proteins and gene expression levels are coupled in Drosophila and are independently associated with mRNA abundance, protein length, and number of protein-protein interactions. Mol Biol Evol. 2005;22:1345-54.

53. Artieri CG, Haerty W, Singh RS. Association between levels of coding sequence divergence and gene misregulation in Drosophila male hybrids. J Mol Evol. 2007:65:697-704.

54. Nuzhdin SV, Wayne ML, Harmon KL, Mclntyre LM. Common pattern of evolution of gene expression level and protein sequence in Drosophila. Mol Biol Evol. 2004;21:1308-17.

55. Castillo-Davis Cl, Hartl DL, Achaz G. cis-Regulatory and protein evolution in orthologous and duplicate genes. Genome Res. 2004;14:1530-6.

56. Go AC, Civetta A. Hybrid incompatibilities and transgressive gene expression between two closely related subspecies of Drosophila. Front Genet. 2020;11:1544.

57. Barreto FS, Pereira RJ, Burton RS. Hybrid dysfunction and physiological compensation in gene expression. Mol Biol Evol. 2015;32:613-22.

58. Patlar B, Civetta A. Speciation and changes in male gene expression in Drosophila. Genome. 2021;64:63-73.

59. Nicolas G. Immediate and latent effects of carbon dioxide on insects. Annu Rev Entomol. 1989;34:97-116.

60. Fiumera AC, Dumont BL, Clark AG. Associations between sperm competition and natural variation in male reproductive genes on the third chromosome of Drosophila melanogaster. Genetics. 2007;176:1245-60.

61. Clark AG, Aguade M, Prout T, Harshman LG, Langley CH. Variation in sperm displacement and its association with accessory gland protein loci in Drosophila melanogaster. Genetics. 1995;139:189-201.

62. Zhang R, Clark AG, Fiumera AC. Natural genetic variation in male reproductive genes contributes to nontransitivity of sperm competitive ability in Drosophila melanogaster. Mol Ecol. 2013;22:1400-15.

63. Fiumera AC, Dumont BL, Clark AG. Sperm competitive ability in Drosophila melanogaster associated with variation in male reproductive proteins. Genetics. 2005;169:243-57.

64. Findlay GD, Sitnik JL, Wang W, Aquadro CF, Clark NL, Wolfner MF. Evolutionary rate covariation identifies new members of a protein network 
required for Drosophila melanogaster female post-mating responses. PLoS Genet. 2014;10:e1004108.

65. Singh A, Buehner NA, Lin H, Baranowski KJ, Findlay GD, Wolfner MF. Longterm interaction between Drosophila sperm and sex peptide is mediated by other seminal proteins that bind only transiently to sperm. Insect Biochem Mol Biol. 2018;102:43-51.

66. LaFlamme BA, Ravi Ram K, Wolfner MF. The Drosophila melanogaster seminal fluid protease "Seminase" regulates proteolytic and post-mating reproductive processes. PLoS Genet. 2012;8:30-2.

67. Kondo S, Vedanayagam J, Mohammed J, Eizadshenass S, Kan L, Pang N, et al. New genes often acquire male specific functions but rarely become essential in Drosophila. Genes Dev. 2017;31:1841-6.

68. Mueller $\mathrm{J}$, Page $\mathrm{L}$, Wolfner MF. An ectopic expression screen reveals the protective and toxic effects of Drosophila seminal fluid proteins. Genetics. 2007;175:777-83.

69. Hopkins BR, Sepil I, Bonham S, Miller T, Charles PD, Fischer R, et al. BMP signaling inhibition in Drosophila secondary cells remodels the seminal proteome and self and rival ejaculate functions. Proc Natl Acad Sci USA. 2019;116:24719-28.

70. Ayroles JF, Laflamme BA, Stone EA, Wolfner MF, Mackay TFC. Functional genome annotation of Drosophila seminal fluid proteins using transcriptional genetic networks. Genet Res (Camb). 2011;93:387-95.

71. Hopkins BR, Sepil I, Thézénas ML, Craig JF, Miller T, Charles PD, et al. Divergent allocation of sperm and the seminal proteome along a competition gradient in Drosophila melanogaster. Proc Natl Acad Sci USA. 2019;116:17925-33.

72. Wong $\mathrm{CL}$, Chan OC, Lee KH, O WS, Chow PH. Absence of paternal accessory sex glands dysregulates preimplantation embryo cell cycle and causes early oviductal-uterine transit in the golden hamster in vivo. Fertil Steril. 2008;89:1021-4.

73. Chapman T, Bangham J, Vinti G, Seifried B, Lung O, Wolfner MF, et al. The sex peptide of Drosophila melanogaster: female post-mating responses analyzed by using RNA interference. Proc Natl Acad Sci USA. 2003;100:9923-8.

74. Grewal G, Patlar B, Civetta A. Expression of Mst89b and CG31287 is needed for effective sperm storage and egg fertilization in Drosophila. Cells. 2021:10:1-12.

75. Schmittgen TD, Livak KJ. Analyzing real-time PCR data by the comparative CT method. Nat Protoc. 2008;3:1101-8.

76. Stewart AD, Hannes AM, Rice WR. An assessment of sperm survival in Drosophila melanogaster. Evolution. 2007;61:636-9.

77. R Development Core Team. R: a language and environment for statistical computing. Vienna, Austria. 2017.

78. Reinhart M, Carney T, Clark AG, Fiumera AC. Characterizing male-female interactions using natural genetic variation in Drosophila melanogaster. Hered. 2015;106(1):67-79.

79. Ravi Ram K, Sirot LK, Wolfner MF. Predicted seminal astacin-like protease is required for processing of reproductive proteins in Drosophila melanogaster. Proc Nat Acad Sci 2006;103(49):18674-18679. https://doi.org/ 10.1073/pnas.0606228103.

\section{Publisher's Note}

Springer Nature remains neutral with regard to jurisdictional claims in published maps and institutional affiliations.
Ready to submit your research? Choose BMC and benefit from:

- fast, convenient online submission

- thorough peer review by experienced researchers in your field

- rapid publication on acceptance

- support for research data, including large and complex data types

- gold Open Access which fosters wider collaboration and increased citations

- maximum visibility for your research: over $100 \mathrm{M}$ website views per year

At BMC, research is always in progress.

Learn more biomedcentral.com/submissions 